\title{
Study on the Influence of Slope on Pollutant Diffusion Characteristics during Tunnel Construction Period
}

\author{
Zubin $\mathrm{Ai}^{1}$, Zhongqiang Song ${ }^{2}$, Fan Chen ${ }^{1}$, Song Yang ${ }^{1}$, Zhensheng Cao ${ }^{1}$, Rong $\mathrm{Liu}^{2}{ }^{*}$ \\ 1. POWERCHINA roadbridge Group CO.,LTD, 100048 Beijing, China \\ 2. Chongqing University, 400044 Chongqing, China
}

\begin{abstract}
The relative elevation of the beginning and end of the Jianyuan Expressway exceed 1000m. In order to overcome the impact of the large slope on traffic safety, a spiral tunnel was designed in the middle of the expressway. The tunnel length is $3970 \mathrm{~m}$ and the radius is $710 \sim 730 \mathrm{~m}$ with its slope being up to $2.08 \%$. The occurrence of large slope has a great impact on ventilation and smoke during tunnel construction, but little research has been done before. In this paper, numerical simulation and field test were used to study the effect of slope on the diffusion characteristics of pollutants. It was found that as the slope increased, the wind speed and $\mathrm{CO}$ dilution rate also increased. However, the dust dilution characteristics were different. Especially in the case of the downward tunnel, the effect of wind speed on the sedimentation rate of dust was minimal. Simply increasing the wind speed cannot speed up the dust reduction rate.
\end{abstract}

\section{Introduction}

Along with the continuous development of national policy "the development of the western region in China", a large number of infrastructure construction begin to appear in the western part of China. Due to the mountainous and hilly terrain in western China, a large number of tunnels have appeared in both road construction and railway construction. The tunnel has a good ability to cross the mountains, and greatly shortening the distance between two places, but when facing two places with large elevation difference, the short distance increases the slope. The relative elevation of the beginning and end of the Jianyuan Expressway exceed $1000 \mathrm{~m}$. To overcome the impact of the large longitudinal slope on traffic safety, a spiral tunnel was designed in the middle of the expressway. The tunnel length is $3970 \mathrm{~m}$ and the radius of $710 \sim 730 \mathrm{~m}$ and the slope reached $2.08 \%$.

To explore the diffusion and dilution characteristics of pollutants in tunnels, a large number of scholars have researched on it. Chow [1] applied the CFD method to study the diffusion and distribution of $\mathrm{CO}$ and finally obtained the distribution of CO. Toraño J [2] applied the CFX software to calculate the dust concentration in the fully mechanized excavation face. And combined with the specific position and distribution of the air duct to establish "E-R" and the "E-F" model. It compared and analyzed the distribution of dust concentration in two modes and then compared the calculation with the measured data. Wei N [3] used the discrete phase model in FLUENT software to numerically simulate the distribution of dust during the ventilation process of the excavation face and compared it with the measured values in construction site. Yang Chunli [4] used CFD to simulate the gas distribution in the tunnel space. Gao Jianliang [5] analyzed the relationship between the flow structure and the gas concentration. Liu Zude [6] analyzed the influence of the outlet diameter and wind speed of the air duct on the flow structure of the whole space. Wang Xiaoling [7] studied the distribution characteristics of carbon monoxide gas. Chai Junrui [8] conducted a study on the concentration of carbon monoxide in the process of ventilation and found out the movement of harmful gases inside the tunneling space with time. There are few reports on the influence of slopes on diffusion characteristics and dilution characteristics for tunnel construction pollutants.

The purpose of this paper is to study the diffusion law of dust and CO under the effect of slope. This paper studies the diffusion characteristics of pollutants under the influence of different slopes in the tunnel during the construction period based on the Midecun Tunnel of Jianyuan Expressway. The second part of the paper shows the $\mathrm{CO}$ field test results and the third part shows the dilution and diffusion laws of $\mathrm{CO}$ and dust under different slopes. The fourth part is analysis and conclusion.

\section{Measurement programs}

\subsection{Introduction of Midecun Tunnel}

Midecun Tunnel is located in the Jianshui County, Yunnan Province, China. The tunnel is a two-line split tunnel with a left length of $3830 \mathrm{~m}$ and a right length of 
$3970 \mathrm{~m}$. The height difference between import and export is about $80 \mathrm{~m}$, and the tunnel slope is $-2.08 \%$ and $-2.05 \%$. The maximum buried depth is $366 \mathrm{~m}$. Figure 2.1 is the map of Midecun Tunnel.

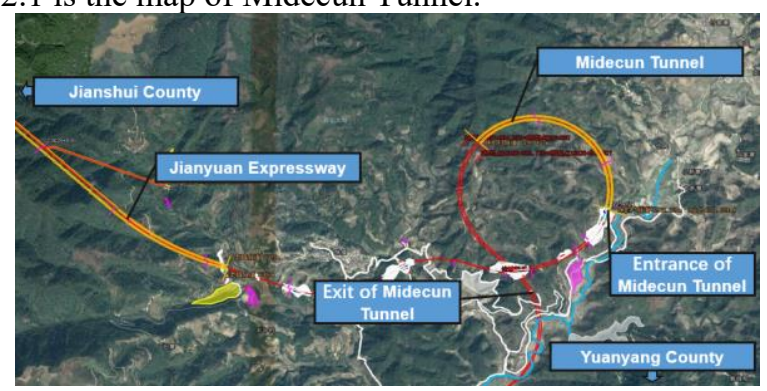

Figure 2.1. Tunnel location map

\subsection{On-site determination of $\mathrm{CO}$ concentration}

The concentration of $\mathrm{CO}$ after blasting was measured on site. The field test after the face blasting and $\mathrm{CO}$ measure point is shown in Figure 2.2. The measuring section $\mathrm{A}, \mathrm{B}$ and $\mathrm{C}$ are three sections from the tunnel face $50 \mathrm{~m}, 100 \mathrm{~m}$ and $150 \mathrm{~m}$ respectively. The point is $1.6 \mathrm{~m}$ height away from the ground. Three monitoring points are arranged at equal distances on the three intersection lines.

In order to ensure safety, the measurement began 1 minutes after the explosion and the $\mathrm{CO}$ concentration was measured every three minutes. Figure 2.3 shows the measured curve of the measured data. It can be seen from the figure that the $\mathrm{CO}$ concentration at different positions shows a trend of increasing and then decreasing. As the distance to the face is getting farther and farther, the maximum concentration of $\mathrm{CO}$ is gradually getting smaller. It can be judged that $\mathrm{CO}$ diffuses as an air mass during diffusion, and the volume of air mass is constantly expanding. Therefore, as the distance of the measuring point increases, the peak value of the $\mathrm{CO}$ concentration gradually decreases. But the range of ultralimit concentration is getting smaller and smaller.

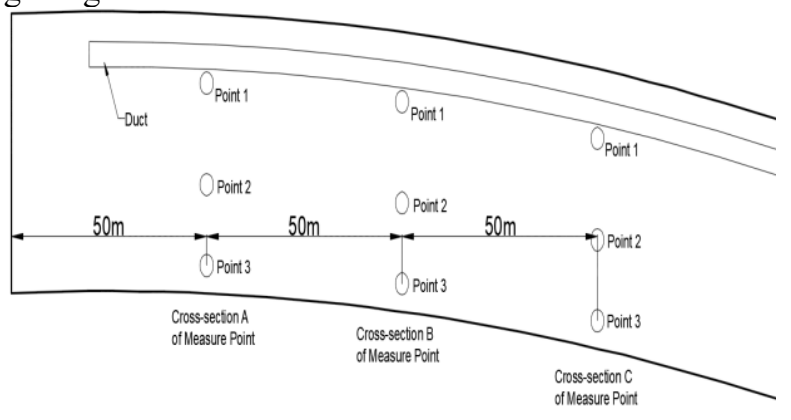

Figure 2.2. Location of measure points

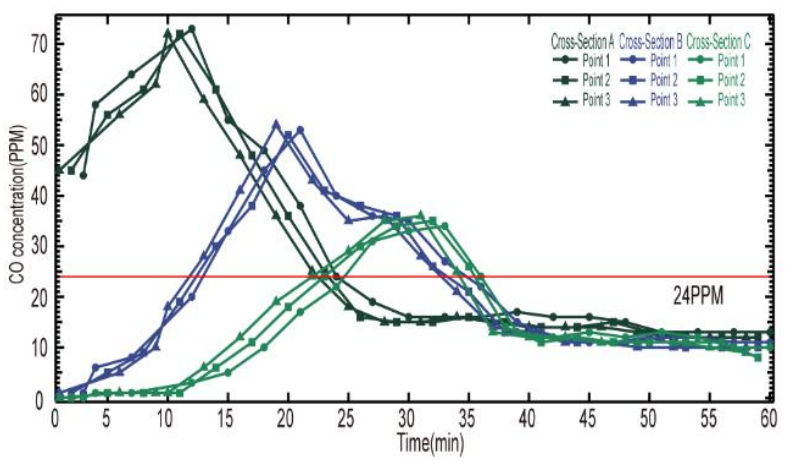

Figure 2.3. CO concentration measured curve

\section{Numerical results and analysis}

\subsection{Introduction to computational model}

The numerical calculation model is established based on the actual engineering situation of Midecun Tunnel. The entrance and exit of tunnel is simultaneously constructed to the tunnel center, so the model is divided into two types: upward spiral and downward spiral. Five gradients of slope are selected for study, and they are $2 \%, 4 \%, 6 \%, 8 \%$ and $10 \%$. The section of the tunnel model is shown in Figure 3.1. The section is obtained by appropriate simplification of the actual tunnel. The air duct diameter is $1.8 \mathrm{~m}$. It is located $3.4 \mathrm{~m}$ away from the ground and $3.45 \mathrm{~m}$ away from the centerline of the tunnel. The outlet of the air duct is $15 \mathrm{~m}$ away from the face. The length of the spiral tunnel model is $300 \mathrm{~m}$. The tunnel geometry model was built by Pro/Engineer and meshed by software ICEM.

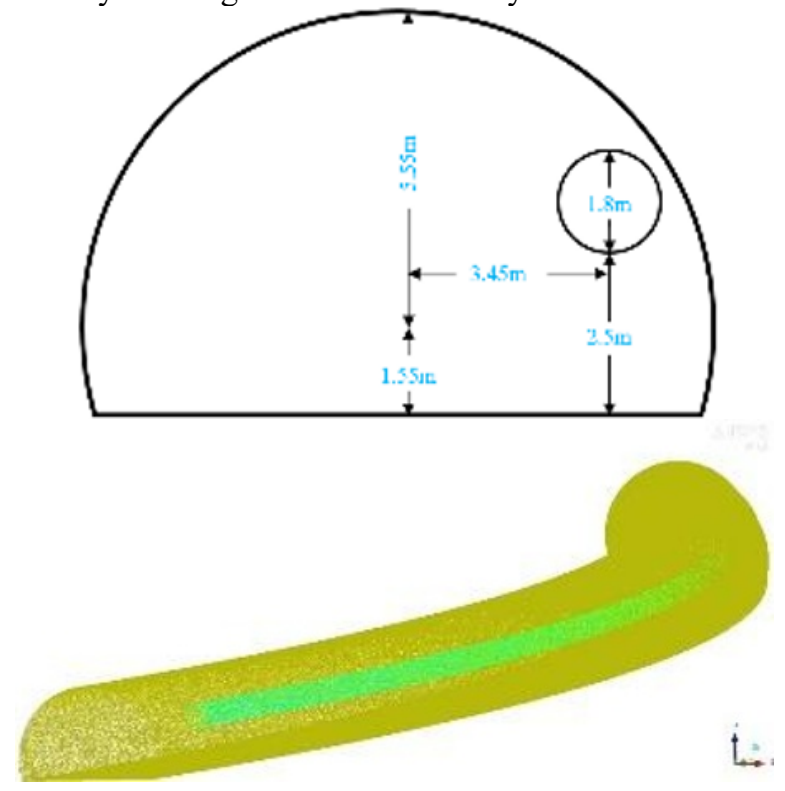

Figure 3. 1. Model profile and model mesh

\subsection{Boundary condition setting}

In the calculation, the tunnel air duct inlet is the flowinlet, $1500 \mathrm{~m}^{3} / \mathrm{min}$, the air duct outlet design value is interior, the dust mass flow rate is $0.04 \mathrm{~kg} / \mathrm{s}$, the 
ground is set to trap, the vault and the gangs are set to reflect. Before the calculation, set the space from crosssection that $60 \mathrm{~m}$ away from the face to the tunnel face to be filled with $\mathrm{CO}$ at a concentration of $0.003 \%$.

\subsection{Result and validation}

\subsubsection{The influence of slope on the characteristics of air flow structure.}

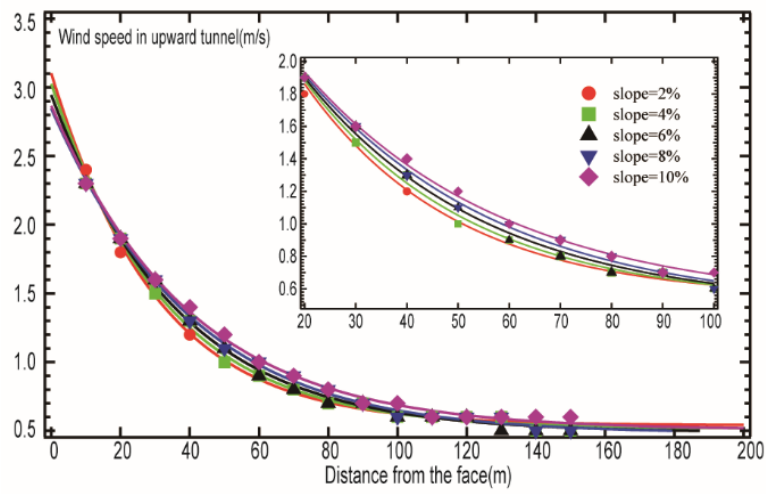

Fig. 3.2 shows the wind speed in the spiral tunnel with different slopes. As the distance from the face increases, the wind speed along the tunnel decreases rapidly, and then turns slowly. Finally the wind speed stabilizes around $0.55 \mathrm{~m} / \mathrm{s}$. By comparing the curve of wind speed with different slopes in the figure, it can be found that the wind speed decreases more slowly as the slope increases. In addition, under the same supply air volume, the overall wind speed of the upward tunnel is smaller than the downward tunnel. This means that the flow in tunnel is more likely to spread upward, but less likely to spread downward.

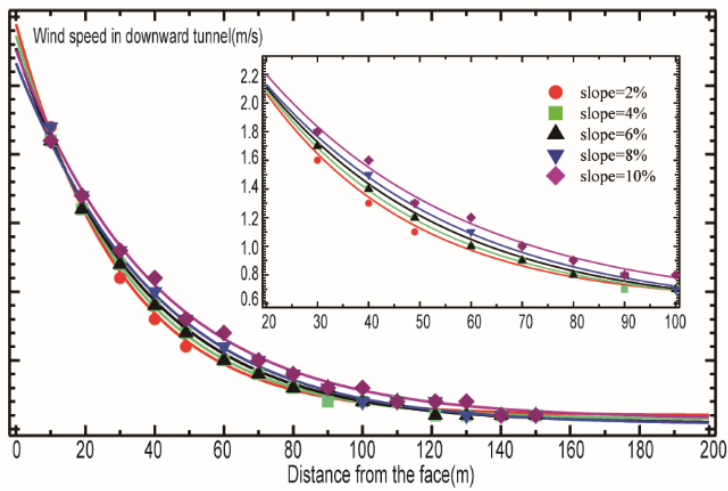

Figure 3.2. Wind speed in spiral tunnel with different slopes

\subsubsection{The influence of slope on the Dust diffusion characteristic.}

Figure 3.3 shows the variation of dust concentration in the tunnel under different slopes. It can be seen from the figure that the influence of slope on the dust concentration shows a different trend in upward tunnel and downward. In upward tunnel, the dust concentration decreases more quickly with larger slope. But in downward tunnel, with larger slope, the dust concentration decreases more slowly. Therefore, the

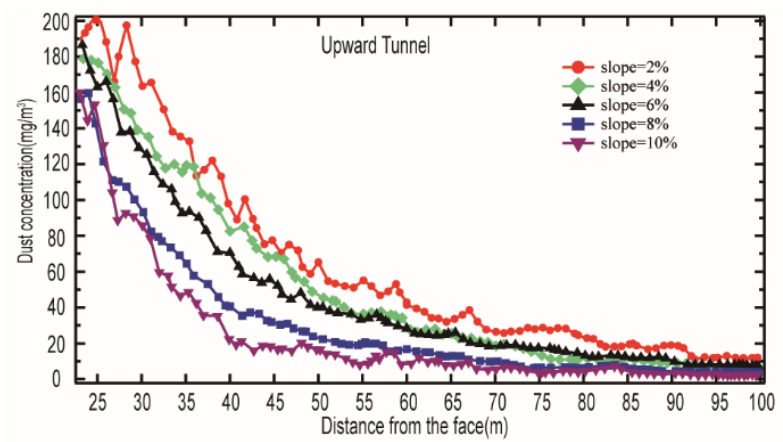

variation trend of dust is different in different construction directions.

Compared with Figure 3.2, it can be found that the variation of dust concentration is less correlated with wind speed. In upward tunnel, wind speed increases with the increase of slope, and dust concentration reduction rate decreases with the increase of slope. In downward tunnel, wind speed increases with the increase of slope, but dust concentration reduction rate decreases with the increase of slope.

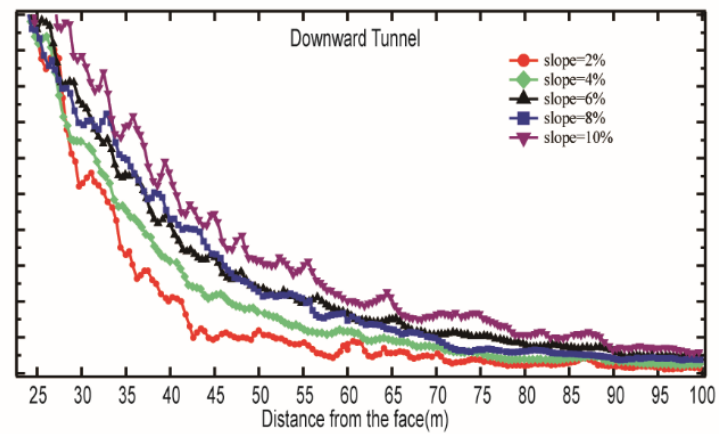

Figure 3.3. Dust concentration in spiral tunnel with different slopes

\subsubsection{The influence of slope on the CO diffusion characteristic.}

The central point which in the section $150 \mathrm{~m}$ away from the face and at $1.6 \mathrm{~m}$ height from the ground is selected as the $\mathrm{CO}$ concentration measurement point, and draw Figure 3.4. No matter what kind of conditions, the $\mathrm{CO}$ concentration at the measurement point has experienced a rapid increase and then decrease quickly. Moreover, the trend of the peak concentration of $\mathrm{CO}$ is the same whether it is the upward tunnel or the downward tunnel. It can be seen that as the slope increases, the peak time of CO concentration is getting shorter and shorter. And the maximum value of the 
peak becomes smaller. The main reason for this law is the effect of wind speed. As the slope increases, the wind speed at the same position increases. Therefore, the $\mathrm{CO}$ dilution rate is accelerated. In the downward tunnel, the $\mathrm{CO}$ concentration is lower than the $\mathrm{CO}$ concentration in the upward tunnel. Also because the wind speed in the downward tunnel is larger than the wind speed in the upward tunnel. Comparing the numerical calculation results and the measured results,
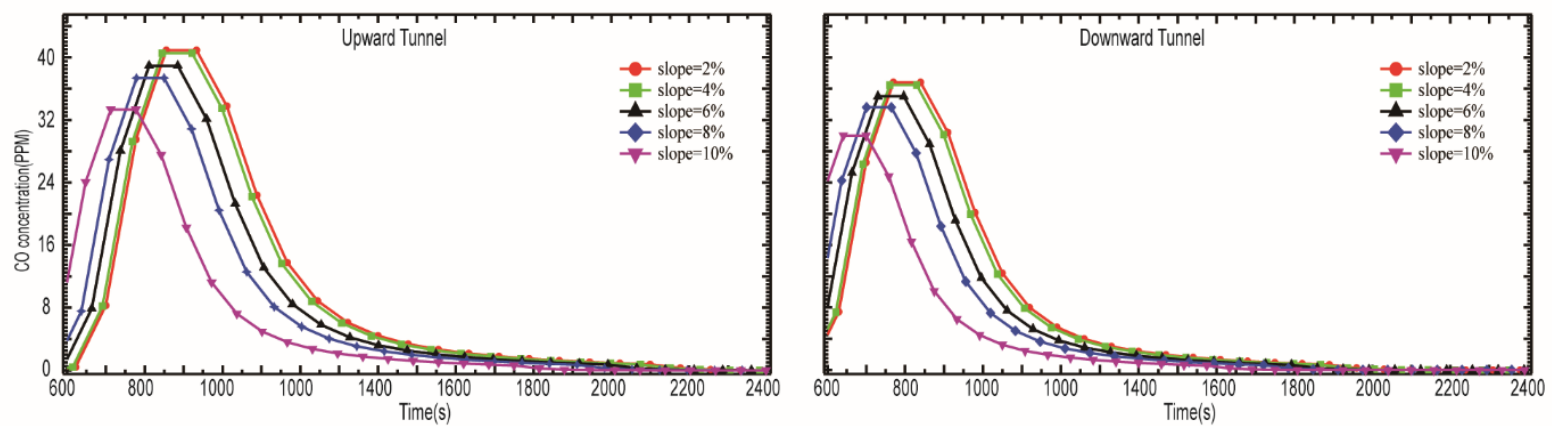

Figure 3.4. CO concentration in spiral tunnel with different slopes

\section{Conclusions}

In this paper, the flow structure characteristics of spiral tunnel with different slopes are studied. It is found that the wind speed in the tunnel decreases rapidly around the tunnel face and then decreases slowly. With the increase of the slope, the change of the wind speed in the spiral tunnel is slowed down, and he wind speed at the same location increases. It shows that the increase of the slope is conducive to the backflow of ventilation in the construction of the spiral tunnel. In addition, the study found that wind speed has little effect on dust diffusion, and the slope has a greater impact on the sedimentation rate of dust. In the upward tunnel, as the slope increases, the wind speed increases and the dust reduction rate also increases. However, in the downward tunnel, as the slope increase, the wind speed increases but dust reduction rate decrease. From the calculation results, the more important factor affecting the diffusion and dilution of $\mathrm{CO}$ is the numerical value of the wind speed.

After the blasting operation, there will be dust generation and toxic and harmful gases such as $\mathrm{CO}$ and $\mathrm{NO}_{\mathrm{x}}$. In upward tunnel, dust and toxic and harmful gases in the tunnel can be exhausted by increasing the air volume. But in downward tunnel, the wind speed has little effect on dust reduction. However, reducing the wind speed will cause the toxic and harmful gases to not be diluted properly. Therefore, in this case, there is a need for a method that can be used for blasting dust reduction. For example, using water method during blasting to reduce dust.

\section{References}

1. W Chow. Tunn Undergr Sp Tech. Disperion of Carbon Monoxide from aVehicular Tunnell the the $\mathrm{CO}$ concentration diffusion trends are the same but the increase rate and the dilution rate are quite different. The main reason is that the air leakage rate of the air duct at the tunnel is high, and the air outlet of the air duct cannot output the same air volume as numeral calculations.
Exit Located along a Hillside. 4,2 (1989).

2. J.Toraño, S.TornoM.Menéndez, M.Gent. Tunn Undergr Sp Tech. Auxiliary ventilation in mining roadways driven with roadheaders: Validated CFD modelling of dust behaviour. 26, 1 (2011).

3. W Niu, Z Jiang, D Tian. Min Sci Tech. Numerical simulation of the factors influencing dust in drilling tunnels: Its application. 21,1 (2011).

4. C Yang, Q Liu, C Yang. J Xi'an Uui Sci Tech.Numerical Simulation of Gas Movement Law in One-head Tunnel. 31,06 (2011).

5. J Gao, W Wu, W Luo. J Safety and Environ. Influence of outlet position of air duct on gas distribution in heading face. 9,01 (2009).

6. J Gao, S Zhang. China Safety Sci J. Numerical Simulation of Airflow Distribution in Pressed Local Ventilation Face. 14,1 (2004).

7. J Zhang, X Wang, H Chen , X Liu, Y Sun. J Hydroelectric Eng .Simulation of airflow and dust diffusion in the heading tunnel of the diversion tunnel. 27,1 (2008).

8. Y Liu, J Chai, X Jia, L Qin, X Sun. Rock \& Soil Mech. Numerical Simulation of Harmful Gas Concentration Diffusion in Pressed Ventilation Tunneling Surfac. 30,2 (2009). 